\title{
STRESS ANALYSIS OF ANISOTROPIC ELASTIC V-NOTCHED BODIES
}

\author{
KUANG-CHONG WU and CHIUN-TSAN CHEN \\ Institute of Applied Mechanics, National Taiwan University, Taipei 106, Taiwan
}

(Received 19 May 1995; in revised form 18 July 1995)

\begin{abstract}
A method to determine singular stress fields near a V-notch in an anisotropic plate is presented. The method is based on a new boundary element formulation recently proposed by $\mathrm{Wu}$ et al. [J. Appl. Mech. 59, 344-348 (1992)] coupled with path-independent integrals relating the neartip field to the far field. Numerical examples are given for plates containing a single edge crack, two collinear edge cracks, an internal crack, a single notch, and two symmetric notches under uniform stress. Copyright (C) 1996 Elsevier Science Ltd.
\end{abstract}

\section{INTRODUCTION}

The presence of V-notches in an elastic body may induce stress singularities at the tips within the context of small strain elasticity theory. Fracture is often initiated in the highlystressed regions near the notch tips. It is thus important to determine the near-tip fields accurately to asses the integrity of notched bodies. With given notch angle, boundary conditions on the notch faces and elasticity moduli, the structure of the singular fields can be determined by asymptotic analysis to within multiplicative constants (Ting and Chou, 1981 ; Wu and Chang, 1993). These multiplcative constants depend on the actual geometry and loading in the problem of interest, in addition to those factors just mentioned. The complete singular stress field is derived by Wu and Chang (1993) for an infinite wedge in the presence of body forces or dislocations. For bodies with finite dimensions, for which analytic solutions are generally not available, near-tip stresses can only be determined by numerical methods.

Due to the singular nature of the stress field, it is usually difficult to calculate accurately stresses near notch tips using regular boundary element or finite element methods. Several techniques have been proposed to overcome the difficulty. For example, eigenfunction expansion method (Gross and Mendelson, 1972), path-independent integral in the case of a crack (Wu, 1989), and a combination of eigenfunction expansion method and standard boundary element method (Barone and Robinson, 1972). In the method of Barone and Robinson, the boundary points are divided into regular points, where the tangents are continuous, and irregular points, where the tangents are not uniquely defined. Eigenfunction expansions are employed to represent the fields on boundary elements connected at irregular boundary points. The system of algebraic equations for solving the boundary displacements at regular points and the multiplicative constants in the eigenfunction expansion are provided by the standard boundary integral equations and path-independent integral representations for the multiplicative constants. Barone and Robinson (1972) only treated isotropic plates.

In this paper the treatment of Barone and Robinson is extended to anisotropic Vnotched bodies. However, instead of the standard boundary element method, a new formulation proposed by Wu et al. (1992) is used. For traction problems, the new boundary element method contains displacement tangential derivatives on the boundary as basic unknowns instead of boundary displacements in the standard method. The new method has several advantages over the standard boundary element method. First, the rigid body translation is eliminated. Second, highcr-order representation for displacements is achieved using the same shape functions. Third, all stress components can be obtained on the boundary without numerical differentiation. Fourth, a well-conditioned system of algebraic 
equations can be obtained regardless of the types of the boundary conditions. Based on Stroh's formalism for anisotropic elasticity, complete fields near the tip of a V-notch in an anisotropic body are derived. Path-independent integrals relating the near-tip fields to fields far removed from the notch tip are obtained.

The plan of the paper is as follows. First, Stroh's formalism for anisotropic elasticity is outlined. Next, the new boundary integral equations are introduced. The near-tip fields and associated path-independent integrals are derived in Section 4. Numerical implementation is discussed in Section 5 where some numerical examples are also presented. Finally some concluding remarks are made.

\section{STROH FORMALISM}

In the following discussion vectors or matrices are denoted by bold-faced symbols. With respect to a rectangular coordinate system $x_{i}(i=1,2,3)$, the generalized Hookes law and the equations of equilibrium are given as

$$
\begin{aligned}
\sigma_{i j} & =C_{i j k l} u_{k, l} \\
\sigma_{i, j,} & =0
\end{aligned}
$$

where $u_{k}$ and $\sigma_{i j}$ are the displacement and stress, respectively, $C_{i j k l}$ is the elasticity tensor and comma denotes partial differentiation. In eqns (1) and (2) repeated indices are summed from 1 to 3 . For deformation in which $u_{k}$ depends only on $x_{1}$ and $x_{2}$, a general solutions to eqn (2) is (Stroh, 1958) :

$$
\mathbf{u}=\mathbf{a} f(z)
$$

where $\mathbf{u}$ is the displacement vector, $z=x_{1}+p x_{2}, p$ and a are a constant scalar and vector, respectively, and $f$ is an arbitrary function of $z$. Substitution of eqn (3) into eqns (1) and (2) leads to

$$
\left[\mathbf{Q}+\left(\mathbf{R}+\mathbf{R}^{\mathrm{T}}\right) p+\mathbf{T} p^{2}\right] \mathbf{a}-0
$$

where the matrices $\mathbf{Q}, \mathbf{R}$, and $\mathbf{T}$ are defined as

$$
\begin{aligned}
Q_{i k} & =C_{i 1 k 1} \\
R_{i k} & =C_{i 1 k 2} \\
T_{i k} & =C_{12 k 2}
\end{aligned}
$$

and superscript $T$ denotes matrix transpose. For nontrivial solutions of $\mathbf{a}$ in eqn (4), wc must have

$$
\operatorname{det}\left[\mathbf{Q}+\left(\mathbf{R}+\mathbf{R}^{\mathrm{T}}\right) p+\mathbf{T} p^{2}\right]=0 .
$$

Substituting eqn (3) into eqn (1), we can express the stress as

$$
\sigma_{i 1}=-\phi_{i, 2}, \quad \sigma_{i 2}=\phi_{i, 1}
$$

where the stress function $\phi$ is given as :

$$
\phi-\mathbf{b} f(z)
$$

and the vector $\mathbf{b}$ is defined as 


$$
\mathbf{b}=\left(\mathbf{R}^{\mathrm{T}}+p \mathbf{T}\right) \mathbf{a}=-\frac{1}{p}(\mathbf{Q}+p \mathbf{R}) \mathbf{a} .
$$

The second identity in eqn (8) is a result of eqn (4). From eqn (6), the traction $t$ on the contour with outward normal $\mathbf{n}$ can be expressed as

$$
\mathbf{t}=\frac{-\partial \phi}{\partial s}
$$

where $s$ is the arc length increasing in the direction obtained by rotating $\mathbf{n}$ counter-clockwise by $\pi / 2$.

Equation (5) provides six roots, $p_{\kappa}, \kappa=1, \ldots, 6$. Since $p_{\kappa}$ are complex if the strain energy is positive definite (Eshelby et al., 1953), we can let

$$
\begin{array}{ll}
p_{\kappa+3}=\bar{p}_{\kappa}, & \mathscr{I}\left[p_{\kappa}\right]>0 \\
\mathbf{a}_{\kappa+3}=\overline{\mathbf{a}}_{\kappa}, & \mathbf{b}_{\kappa+3}=\overline{\mathbf{b}}_{\kappa}, \quad \kappa=1,2,3 .
\end{array}
$$

where $\mathscr{I}$ denotes imaginary part and overbar stands for complex conjugate. If the roots $p_{\kappa}$ are distinct, the general expressions for the displacement $\mathbf{u}$ and stress function $\phi$ given by eqn (3) and eqn (7), respectively, can be written as

$$
\begin{aligned}
& \mathbf{u}=\sum_{\kappa=1}^{6} \mathbf{a}_{\kappa} f_{\kappa}\left(z_{\kappa}\right), \\
& \phi=\sum_{\kappa=1}^{6} \mathbf{b}_{\kappa} f_{\kappa}\left(z_{\kappa}\right),
\end{aligned}
$$

where $f_{\kappa}$ are arbitrary functions of their arguments.

The two equations in eqn (8) can be rearranged into the following form

$$
\mathbf{N} \xi=p \xi
$$

where

$$
\begin{aligned}
& \mathbf{N}=\left(\begin{array}{ll}
\mathbf{N}_{1} & \mathbf{N}_{2} \\
\mathbf{N}_{3} & \mathbf{N}_{1}^{\mathrm{T}}
\end{array}\right) \\
& \boldsymbol{\xi}=\left(\begin{array}{l}
\mathbf{a} \\
\mathbf{b}
\end{array}\right)
\end{aligned}
$$

and

$$
\begin{aligned}
& \mathbf{N}_{1}=-\mathbf{T}^{-1} \mathbf{R}^{\mathrm{T}} \\
& \mathbf{N}_{2}=\mathbf{T}^{-1}=\mathbf{N}_{2}^{\mathrm{T}} \\
& \mathbf{N}_{3}=\mathbf{R} \mathbf{T}^{-1} \mathbf{R}^{\mathrm{T}}-\mathbf{Q} .
\end{aligned}
$$

Let $\boldsymbol{\eta}$ be the left eigenvector of $\mathbf{N}$ determined by

$$
\mathbf{N}^{\mathrm{T}} \boldsymbol{\eta}=\boldsymbol{p} \boldsymbol{\eta}
$$




$$
\mathbf{N}^{\top}=\mathbf{J N} \mathbf{J}
$$

where

$$
\mathbf{I}=\left(\begin{array}{ll}
\mathbf{0} & \mathbf{I} \\
\mathbf{I} & \mathbf{0}
\end{array}\right)
$$

I being the $3 \times 3$ identity matrix. Comparing eqn (13) with eqn (12), we can set

$$
\boldsymbol{\eta}=\mathbf{J} \xi=\left(\begin{array}{l}
\mathbf{b} \\
\mathbf{a}
\end{array}\right)
$$

As the left eigenvector $\boldsymbol{\eta}$ and the right eigenvector $\xi$ associated with different eigenvalue of $p$ are bi-orthogonal to each other, we can normalize the eigenvectors such that

$$
\boldsymbol{\eta}_{k}^{\mathrm{T}} \xi_{;}=\dot{\delta}_{\kappa ;} \quad \text { or } \mathbf{a}_{\kappa}^{\mathrm{T}} \mathbf{b}_{;}+\mathbf{b}_{\kappa}^{\mathrm{T}} \mathbf{a}_{i}=\delta_{\kappa ;},
$$

where $\delta_{n \gamma}$ is Kronecker's delta. On account of eqn (15) the $6 \times 6$ unit matrix $\mathbf{I}_{6}$ can be represented as

$$
\mathbf{I}_{h}=\sum_{n=1}^{h} \boldsymbol{\xi}_{k} \boldsymbol{\eta}_{k}^{\top}
$$

or

$$
\begin{aligned}
& \sum_{\kappa=1}^{6} \mathbf{a}_{k} \mathbf{a}_{k}^{\mathrm{T}}=2 \mathscr{R}\left(\sum_{k=1}^{6} \mathbf{a}_{k} \mathbf{a}_{k}^{\mathrm{T}}\right)=\mathbf{0}, \\
& \sum_{n=1}^{6} \mathbf{b}_{n} \mathbf{b}_{n}^{T}=2 \mathscr{R}\left(\sum_{k=1}^{6} \mathbf{b}_{h} \mathbf{b}_{k}^{T}\right)=\mathbf{0}, \\
& \sum_{k=1}^{6} \mathbf{a}_{k} \mathbf{b}_{k}^{\mathrm{T}}=2 \mathscr{R}\left(\sum_{k=1}^{6} \mathbf{a}_{k} \mathbf{b}_{k}^{\mathrm{T}}\right)=\mathbf{I},
\end{aligned}
$$

where $\mathscr{R}$ denotes real part. With eqns $(10),(11)$ and $(15)$, the function $f_{\kappa}\left(z_{\kappa}\right)$ can be expressed as

$$
f_{n}\left(z_{n}\right)=\mathbf{a}_{\wedge}^{\mathrm{T}} \boldsymbol{\phi}+\mathbf{b}_{n}^{\mathrm{T}} \mathbf{u}
$$

\section{DUAL BOUNDARY INTEGRAL EQUATIONS}

The conventional boundary integral equation formulation was first developed by Rizzo (1967) for two-dimensional linear elasticity problems. The formulation is derived by applying Betti's reciprocal theorem to the elastic state of interest and the auxiliary state due to a body force. In the conventional formulation the boundary integral equations are expressed in terms of the displacements and tractions on the boundary. In this section a new boundary integral equation formulation proposed by Wu et al. (1992) based on distributions of body forces and dislocation is introduced. The new formulation consists of dual sets of boundary integral equations involving the tangential gradient of the displacements and tractions on the boundary. Here we show that the integral equations are a direct consequence of analyticity of complex functions in Stroh's formalism.

In the theory of complex analytic functions it is well known that a function $f(z)$ of $z=x_{1}+i x_{2}, i=\sqrt{-1}$, analytic in a closed region $R$ can be expressed in terms of its values on the boundary $C$ of $R$ by Cauchy's integral formula as 


$$
f(z)=\frac{1}{2 \pi \mathrm{i}} \int_{C} \frac{f\left(z^{\prime}\right)}{z^{\prime}-z} \mathrm{~d} z^{\prime}
$$

where the direction of integration is counter-clockwise. Equation (21) can be extended to analytic functions $f_{\kappa}\left(z_{\kappa}\right)$ in Stroh's formalism, since we can regard the complex variables $z_{\kappa}=x_{1}+p_{\kappa} x_{2}$ as $z_{\kappa}=y_{1}+\mathrm{i} y_{2}$, where $y_{1}=x_{1}+\mathscr{R}[p] x_{2}$ and $y_{2}=\mathscr{I}\left[p_{\kappa}\right] x_{2}$. The direction of intcgration in cquation (21) for $f_{\kappa}\left(z_{\kappa}\right)$ however depends on the $\mathscr{I}\left[p_{\kappa}\right]$. For $\kappa=1,2,3$, $\mathscr{I}\left[p_{\kappa}\right]>0$, the direction of integration in eqn (21) is counter-clockwise whereas for $\kappa=4$, $5,6, \mathscr{I}\left[p_{\kappa}\right]<0$, the direction of integration is clockwise. Thus the derivative of $f_{\kappa}$ with respect to $z_{\kappa}, \mathrm{d} f_{\kappa} / \mathrm{d} z_{\kappa}, \kappa=1,2,3$, which are also analytic, can be expressed as

$$
\frac{\mathrm{d} f\left(z_{\kappa}\right)}{\mathrm{d} z_{\kappa}}=\frac{1}{2 \pi \mathrm{i}} \int_{C} \frac{1}{z_{\kappa}^{\prime}-z_{\kappa}} \frac{\mathrm{d} f\left(z_{\kappa}^{\prime}\right)}{\mathrm{d} z_{\kappa}^{\prime}} \mathrm{d} z_{\kappa}^{\prime} .
$$

The limiting form of eqn (22) as a smooth boundary point is approached becomes

$$
\frac{\mathrm{d} f\left(z_{\kappa}\right)}{\mathrm{d} z_{\kappa}}=\frac{1}{\pi \mathrm{i}} \int_{C} \frac{1}{z_{\kappa}^{\prime}-z_{\kappa}} \frac{\mathrm{d} f\left(z_{\kappa}^{\prime}\right)}{\mathrm{d} z_{\kappa}^{\prime}} \mathrm{d} z_{\kappa}^{\prime} .
$$

From eqn (20), the tangential gradient of $f_{\kappa}$ can be expressed as

$$
\frac{\partial f_{\kappa}}{\partial s}=-\mathbf{a}_{\kappa}^{\mathrm{T}} \mathbf{t}+\mathbf{b}_{\kappa}^{\mathrm{T}} \mathbf{d}=\frac{\mathrm{d} f\left(z_{\kappa}\right)}{\mathrm{d} z_{\kappa}} \frac{\partial z_{\kappa}}{\partial s}
$$

where $s$ is the arc length of the boundary, $t$ is the traction given by eqn (9), and $d$ is the tangential gradient of the displacement given by

$$
\mathbf{d}=\frac{\partial \mathbf{u}}{\partial \mathbf{s}}
$$

Substitution of eqn (23) into eqn (24) leads to

$$
-\mathbf{a}_{\kappa}^{\mathrm{T}} \mathbf{t}+\mathbf{b}_{\kappa}^{\mathrm{T}} \mathbf{d}=\frac{1}{\pi \mathrm{i}} \frac{\partial z_{\kappa}}{\partial s} \int_{C} \frac{1}{z_{\kappa}^{\prime}-z_{\kappa}}\left(-\mathbf{a}_{\kappa}^{\mathrm{T}} \mathbf{t}+\mathbf{b}_{\kappa}^{\mathrm{T}} \mathbf{d}\right) \mathrm{d} s^{\prime} .
$$

From eqns (17)-(19), we can express $d$ and $t$ as

$$
\begin{aligned}
& \frac{\mathbf{d}}{\mathbf{2}}=\mathscr{R}\left[\sum_{\kappa=1}^{3} \mathbf{a}_{\kappa}\left(-\mathbf{a}_{\kappa}^{\mathrm{T}} \mathbf{t}+\mathbf{b}_{\kappa}^{\mathrm{T}} \mathbf{d}\right)\right], \\
& \frac{\mathbf{t}}{\mathbf{2}}=-\mathscr{R}\left[\sum_{\kappa=1}^{3} \mathbf{b}_{\kappa}\left(-\mathbf{a}_{\kappa}^{\mathrm{T}} \mathbf{t}+\mathbf{b}_{\kappa}^{\mathrm{T}} \mathbf{d}\right)\right] .
\end{aligned}
$$

Substitution of eqn (26) into eqns (27) and (28) yields

$$
\begin{aligned}
& \frac{\mathbf{d}}{2}=\int_{c}(\hat{\mathbf{U}} \mathbf{t}-\hat{\mathbf{W}} \mathbf{d}) \mathrm{d} s \\
& \frac{\mathbf{t}}{2}=\int_{c}\left(-\hat{\mathbf{W}}^{\mathrm{T}} \mathbf{t}+\hat{\mathbf{V}} \mathbf{d}\right) \mathrm{d} s,
\end{aligned}
$$

where the matrices $\hat{\mathbf{U}}, \hat{\mathbf{U}}$, and $\hat{\mathbf{W}}$ are given as : 


$$
\begin{aligned}
& \hat{\mathbf{U}}=-\frac{1}{\pi} \mathscr{I}\left[\sum_{\kappa=1}^{3} \frac{\partial z_{\kappa}}{\partial s} \frac{1}{z_{\kappa}^{\prime}-z_{\kappa}} \mathbf{a}_{k} \mathbf{a}_{\kappa}^{\top}\right] \\
& \hat{\mathbf{V}}=-\frac{1}{\pi} \mathscr{I}\left[\sum_{\kappa=1}^{3} \frac{\partial z_{\kappa}}{\partial s} \frac{1}{z_{\kappa}^{\prime}-z_{\kappa}} \mathbf{b}_{\kappa} \mathbf{b}_{\kappa}^{\mathrm{T}}\right]
\end{aligned}
$$

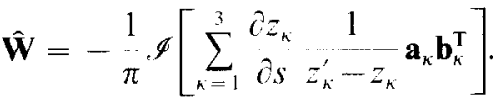

Equations (29) and (30) are dual sets of boundary integral equations for the tangential displacement gradients and tractions. The sets of the boundary integral equations of course are not independent. We therefore have the flexibility to use equations from either set. This leads to a distinct feature of the present formulation that a well-conditioned system of linear algebraic equations can always be established in numerical implementation regardless of types of boundary conditions. The feature will be discussed in Section 5.

\section{NEAR-TIP FIELDS}

The field in the vicinity of a notch of angle $2 \alpha$ can be studied by considering the infinite wedge shown in Fig. 1. The origin is fixed at the notch tip and the $x_{1}$ axis is chosen to coincide with bisector of the wedge such that the upper and lower wedge faces are at $\theta=\alpha$ and $\theta=-\alpha$, respectively, where $\theta$ is the polar angle. The notch faces are assumed traction free. For convenience, $z_{\kappa}$ is expressed as $z_{\kappa}=r \zeta_{\kappa}(\theta)$, where $\zeta_{\kappa}(\theta)=\cos (\theta)+p_{\kappa} \sin (\theta)$ and $r$ is the radial distance from the tip.

Let Stroh's functions introduced in the previous section be of the following form:

$$
f_{k}\left(z_{k}, \lambda\right)=\left[\frac{z_{\kappa}}{\zeta_{k}(-\alpha)}\right]^{\prime} \mathbf{b}_{k}^{\mathrm{T}} \mathbf{q}=r^{j}\left[\frac{\zeta_{\kappa}(\theta)}{\zeta_{\kappa}(-\alpha)}\right]^{\prime} \mathbf{b}_{k}^{\mathrm{T}} \mathbf{q},
$$

where $\lambda$ and $\mathbf{q}$ are a constant scalar and vector, respectively. The displacements and the stress functions obtained by substituting eqn (31) into eqns (10) and (11) are given by

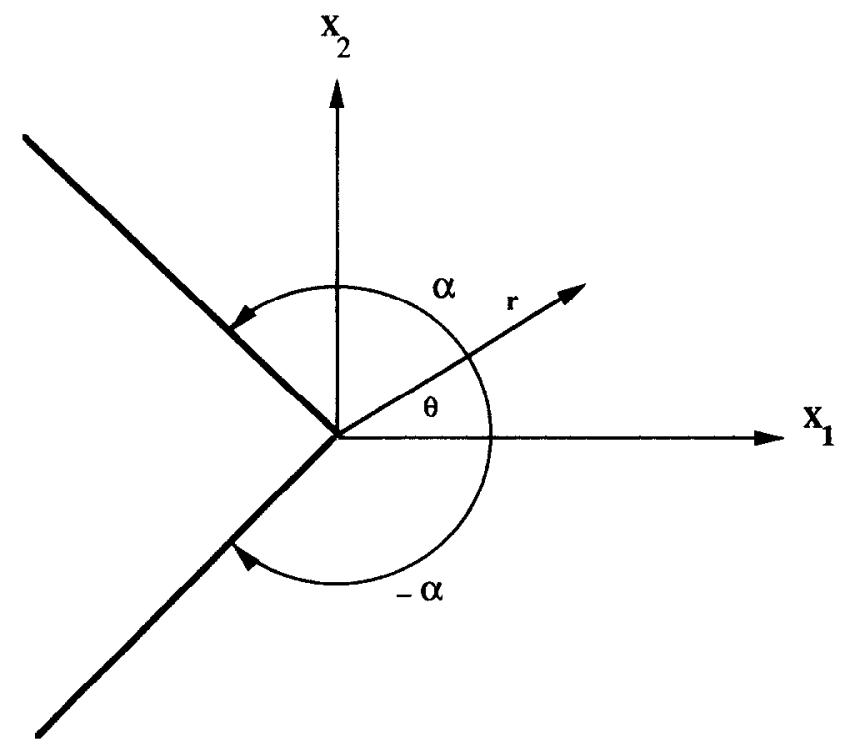

Fig. 1. An infinite wedge of angle $2 \alpha$. 


$$
\begin{aligned}
& \mathbf{u}=r^{\lambda} \sum_{\kappa=1}^{6}\left[\frac{\zeta_{\kappa}(\theta)}{\zeta_{\kappa}(-\alpha)}\right]^{\lambda} \mathbf{a}_{\kappa} \mathbf{b}_{\kappa}^{\mathrm{T}} \mathbf{q}(\lambda), \\
& \boldsymbol{\phi}=r^{\lambda} \sum_{\kappa=1}^{6}\left[\frac{\zeta_{\kappa}(\theta)}{\zeta_{\kappa}(-\alpha)}\right]^{\lambda} \mathbf{b}_{\kappa} \mathbf{b}_{\kappa}^{\mathrm{T}} \mathbf{q}(\lambda) .
\end{aligned}
$$

On account of eqn (18), the traction $t$ corresponding to eqn (33) is identically zero at $\theta=-\alpha$. The requirement that $\mathbf{t}$ vanish at $\theta=\alpha$ then leads to

$$
\lambda \mathbf{D}(\lambda) \mathbf{q}(\lambda)=\mathbf{0},
$$

where $\mathbf{D}(\lambda)$ is a symmetric matrix given by

$$
\mathbf{D}(\lambda)=\sum_{\kappa=1}^{6}\left[\frac{\zeta_{\kappa}(\alpha)}{\zeta_{\kappa}(-\alpha)}\right]^{\lambda} \mathbf{b}_{\kappa} \mathbf{b}_{\kappa}^{\mathbf{T}}
$$

For nontrivial solution of $\mathbf{q}$ we must have

$$
\lambda^{3} \operatorname{det}[\mathbf{D}(\lambda)]=0
$$

Equation (36) is the characteristic equation for $\lambda$. For each root of $\lambda$ a corresponding $q$ is determined by eqn (34) to within a multiplicative constant. For definiteness we let $\mathbf{q}^{\mathrm{T}} \mathbf{q}=1$. Note that if $\lambda$ is complex, $\mathbf{u}$ and $\phi$ given by eqns (32) and (33) are also complex. Since the conjugate of the matrix $\mathbf{D}$ of eqn (35) is given by

$$
\overline{\mathbf{D}(\lambda)}=\mathbf{D}(\bar{\lambda})
$$

if $\lambda$ is a root of eqn (36), so is $\bar{\lambda}$ with the corresponding $\mathbf{q}(\bar{\lambda})=\overline{\mathbf{q}(\lambda)}$. One can superimpose two expressions associated with $\lambda$ and $\bar{\lambda}$ to obtain real $\mathbf{u}$ and $\phi$. As $\mathbf{u}$, given by eqn (32), is proportional to $r^{\lambda}$, we must require $\mathscr{R}[\lambda]>0$ for bounded displacements. If $\mathscr{R}[\lambda]<1$, the corresponding stresses are singular at $r=0$. Assuming that the roots of eqn (36) with positive real parts denoted by $\lambda_{n}, n=1,2, \ldots, \infty$ are distinct, Stroh's function for the complete near-tip fields can be expressed in the following eigenfunction expansion:

$$
f_{\kappa}\left(z_{\kappa}\right)=\sum_{n=1}^{\infty} c_{n} f_{\kappa}\left(z_{\kappa}, \lambda_{n}\right)
$$

where $f_{\kappa}\left(z_{\kappa}, \lambda_{n}\right)$ is given by eqn (31) and $c_{n}$ is a constant.

The roots of eqn (36) have the property that if $\lambda$ is a root so is $-\lambda$. This is proved by defining a vector $\mathbf{q}^{*}$ as

$$
\mathbf{q}^{*}(\lambda)=\left\{\sum_{\kappa=1}^{6}\left[\frac{\zeta_{\kappa}(\alpha)}{\zeta_{\kappa}(-\alpha)}\right]^{\lambda} \mathbf{a}_{\kappa} \mathbf{b}_{\kappa}^{\mathrm{T}}\right\} \mathbf{q}(\lambda)
$$

By using eqns (15) and (34), eqn (39) can be expressed as

$$
\mathbf{b}_{\kappa}^{\mathrm{T}} \mathbf{q}^{*}(\lambda)=\left[\frac{\zeta_{\kappa}(\alpha)}{\zeta_{\kappa}(-\alpha)}\right]^{\lambda} \mathbf{b}_{\kappa}^{\mathrm{T}} \mathbf{q}(\lambda)
$$

Thus 


$$
\mathbf{D}(-\lambda) \mathbf{q}^{*}(\lambda)=\left(\sum_{k=1}^{6} \mathbf{b}_{\kappa} \mathbf{b}_{\kappa}^{\mathbf{T}}\right) \mathbf{q}(\lambda)=\mathbf{0}
$$

In deriving eqn (41), eqns (40) and (18) have been used. Comparing eqn (41) with eqn (34), we conclude that $-\lambda$ is a root and $\mathbf{q}^{*}(\lambda)=\mathbf{q}(-\lambda)$. By eqns (31) and (39), the eigenfunction corresponding to $-\lambda$ can be expressed as

$$
f_{\kappa}\left(z_{\kappa},-\lambda\right)=\left[\frac{z_{\kappa}}{\zeta_{\kappa}(\alpha)}\right]^{-i} \mathbf{b}_{\kappa}^{\mathrm{T}} \mathbf{q}(\lambda)=r^{-\lambda}\left[\frac{\zeta_{\kappa}(\theta)}{\zeta_{\kappa}(\alpha)}\right]^{-i} \mathbf{b}_{\kappa}^{\mathrm{T}} \mathbf{q}(\lambda)
$$

The displacement $\mathbf{u}_{n}^{*}$ and stress function $\boldsymbol{\phi}_{n}^{*}$ obtained by substituting eqn (42) into eqns (10) and (11) with $\lambda=\lambda_{n}$ are

$$
\begin{aligned}
& \mathbf{u}_{n}^{*}=r \lambda_{n} \sum_{k=1}^{6}\left[\begin{array}{l}
\zeta_{\kappa}(\theta) \\
\zeta_{\kappa}(\alpha)
\end{array}\right]^{\cdots \lambda_{n}} \mathbf{a}_{k} \mathbf{b}_{k}^{\mathrm{T}} \mathbf{q}\left(\lambda_{n}\right), \\
& \boldsymbol{\phi}_{n}^{*}=r \sum_{n=1}^{6}\left[\frac{\zeta_{k}(\theta)}{\zeta_{k}(\alpha)}\right]^{\cdots \lambda_{n}} \mathbf{b}_{k} \mathbf{b}_{k}^{\mathrm{T}} \mathbf{q}\left(\lambda_{n}\right) .
\end{aligned}
$$

Let us define an integral $J_{\kappa}$ as

$$
J_{\kappa}=\int_{\gamma} f_{\kappa}\left(z_{\kappa},-\lambda_{m}\right) \frac{\partial f_{\kappa}\left(z_{\kappa}, \lambda_{n}\right)}{\partial z_{\kappa}} \mathrm{d} z_{\kappa}
$$

where $\gamma$ is a contour starting from a point at $r=r_{1}$ on the notch face at $\theta=-\alpha$ to a point at $r=r_{2}$ on the notch face at $\theta=\alpha$. Substitution of eqns (31) and (42) into eqn (45) yields

$$
\begin{aligned}
& J_{\kappa}=\frac{\lambda_{n}}{\lambda_{n}-\lambda_{m}}\left\{r_{2}^{\lambda_{n}^{n}-\lambda_{m}}\left[\frac{\zeta_{\kappa}(\alpha)}{\zeta_{\kappa}(-\alpha)}\right]^{\lambda_{n}}-r_{1^{\prime \prime}}^{\prime^{\prime}}-\lambda_{m}\left[\frac{\zeta_{\kappa}(\alpha)}{\zeta_{\kappa}(-\alpha)}\right]^{\lambda_{m}}\right\} \mathbf{q}_{m}^{1} \mathbf{b}_{\kappa} \mathbf{b}_{\kappa}^{\mathrm{T}} \mathbf{q}_{n}, \quad m \neq n \\
& J_{k}=\lambda_{n} \log \left[\frac{r_{2} \zeta_{\kappa}(\alpha)}{r_{1} \zeta_{\kappa}(-\alpha)}\right]\left[\frac{\zeta_{\kappa}(\alpha)}{\zeta_{\kappa}(-\alpha)}\right]^{\zeta_{n}} \mathbf{q}_{m}^{\mathrm{T}} \mathbf{b}_{\kappa} \mathbf{b}_{\kappa}^{\mathrm{T}} \mathbf{q}_{n}, \quad m=n
\end{aligned}
$$

where $\mathbf{q}_{m}=\mathbf{q}\left(\lambda_{m}\right)$. Since either $\lambda_{m}$ or $\lambda_{n}$ satisfies eqn (34), it follows that

$$
\begin{aligned}
\sum_{\kappa=1}^{6} J_{k} & =\frac{i_{n}}{\lambda_{n}-\lambda_{m}}\left(r_{2^{n}}^{i_{n}-i_{m}} \mathbf{q}_{m}^{\mathrm{T}} \mathbf{D}_{n} \mathbf{q}_{n}-r_{1^{\prime \prime}}^{\lambda^{\prime}-\lambda_{m}} \mathbf{q}_{m}^{\mathrm{T}} \mathbf{D}_{m} \mathbf{q}_{n}\right) \\
& =0, \quad m \neq n \\
\sum_{\kappa=1}^{6} J_{\kappa} & =\lambda_{n}\left[\log \left(\frac{r_{2}}{r_{1}}\right) \mathbf{q}_{n}^{\mathrm{T}} \mathbf{D}_{n} \mathbf{q}_{n}+\mathbf{q}_{n}^{\mathrm{T}} \mathbf{D}_{n}^{\prime} \mathbf{q}_{n}\right]=\delta_{n}^{\prime}, \quad m=n
\end{aligned}
$$

where $\mathbf{D}_{m}=\mathbf{D}\left(\lambda_{m}\right)$ and $\delta_{n}^{\prime}$ is defined as

$$
\check{\delta}_{n}^{\prime}=\lambda_{n} \mathbf{q}_{n}^{\mathrm{T}} \mathbf{D}_{n}^{\prime} \mathbf{q}_{n},
$$

in which $\mathbf{D}_{n}^{\prime}$ is given by 


$$
\mathbf{D}_{n}^{\prime}=\sum_{\kappa=1}^{6} \log \left[\frac{\zeta_{\kappa}(\alpha)}{\zeta_{\kappa}(-\alpha)}\right]\left[\frac{\zeta_{\kappa}(\alpha)}{\zeta_{\kappa}(-\alpha)}\right]^{\lambda_{n}} \mathbf{b}_{\kappa} \mathbf{b}_{\kappa}^{\mathrm{T}}
$$

Note that eqns (48) and (49) do not contain $r_{1}$ or $r_{2}$. Thus $\gamma$ can be any simple contour starting from the notch face $\theta=-\alpha$ and to the notch face $\theta=\alpha$. With eqns (48) and (49) $c_{n}$ in eqn (38) can be represented as

$$
c_{n}=\frac{1}{\delta_{n}^{\prime}} \sum_{\kappa=1}^{6} \int_{\gamma} f_{\kappa}\left(z_{\kappa},-\lambda_{n}\right) \frac{\partial f_{\kappa}\left(z_{\kappa}\right)}{\partial z_{\kappa}} \mathrm{d} z_{\kappa}=\frac{1}{\delta_{n}^{\prime}} \int_{\gamma}\left[\left(\phi_{n}^{*}\right)^{\mathrm{T}} \mathbf{d}-\left(\mathbf{u}_{n}^{*}\right)^{\mathrm{T}} \mathbf{t}\right] \mathrm{d} s .
$$

In deriving the second equation in eqn (52) use has been made of eqn (20) and eqns (17)-(19). Equation (52) is a generalization of the result by $\mathrm{Wu}(1989)$ for stress intensity factors associated with singular crack-tip field. With eqn (52) complete, near-tip fields can be determined by fields far away from the tip.

\section{V-NOTCHED PLATES UNDER TENSION}

Although the analysis discussed in the previous sections is applicable to general anisotropic materials, we restrict our attention to orthotropic materials with symmetry axes parallel to the coordinate axes. For orthotropic materials, the displacements $u_{1}$ and $u_{2}$ in plane strain deformation and $u_{3}$ in anti-plane strain deformation are uncoupled. Only plane deformation is studied here.

Consider the region $\mathrm{OABCD}$ as shown in Fig. 2 with the boundary conditions $t_{1}=d_{2}=0$ on OA and $t_{1}=0$ and $t_{2}=\sigma_{0}$ on BC. The configuration in Fig. 2 represents one-half or one-quarter of three types of notched plates under uniform tension: (1) a plate of height $2 h$ and width $w$ containing an edge notch of angle $2 \alpha$ and depth $a$ if $t_{1}=t_{2}=0$ are specified on $\mathrm{AB}$ and $\mathrm{CD} ;(2)$ a plate of height $2 h$ and width $2 w$ containing double edge notches of angle $2 \alpha$ and depth $a$ if $d_{1}=t_{2}=0$ on $\mathrm{AB}$ and $t_{1}=t_{2}=0$ on CD; and (3) a plate of height $2 h$ and width $2 w$ containing an internal crack $(\alpha=\pi)$ of length $2 a$ if $t_{1}=t_{2}=0$ on $\mathrm{AB}$ and $d_{1}=t_{2}=0$ on $\mathrm{CD}$.

For the problem of interest, the stresses in the plate depend only on two Stroh's eigenvalues $p_{1}$ and $p_{2}$ (Ting, 1995). In the numerical calculations to be presented, the relevant elasticity constants denoted by contracted notations, $C_{11}, C_{12}, C_{12}$, and $C_{66}$ are assumed to satisfy the following relation:

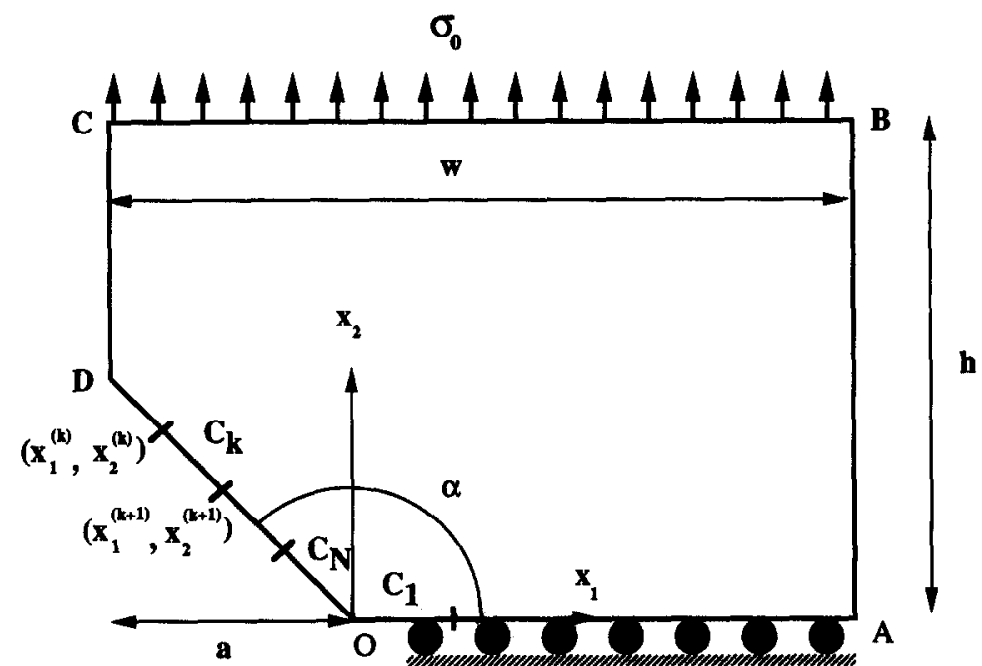

Fig. 2. Configuration considered in the numerical computations. 


$$
C_{11}+2 C_{12}+C_{22}=\frac{C_{11} C_{22}-C_{12}^{2}}{C_{66}} .
$$

For such class of materials, the Stroh eigenvalues $p_{1}$ and $p_{2}$ are given by $p_{1}=\mathrm{i}$ and $p_{2}=\mathrm{i} \beta$, where $\beta=\sqrt{C_{11} / C_{22}}$ (Dongye and Ting, 1989). Note that isotropic material is included by letting $\beta$ approach to 1 .

For the symmetric plane deformation considered, the characteristic equation of $\lambda$, eqn (36), becomes (Wu and Chang, 1993):

$$
\frac{1}{\beta} \cos \lambda \alpha \sin \lambda \tilde{\alpha}-\sin \lambda \alpha \cos \lambda \tilde{\alpha}=0
$$

where $\tilde{\alpha}=\tan ^{-1}(\beta \alpha)$ for orthotropic material and

$$
i \sin (2 \alpha)+\sin (2 \lambda \alpha)=0
$$

for isotropic material. The eigenvector $\mathbf{q}$ of eqn (35) is given by

$$
\mathbf{q}=(-\cos \lambda \alpha, \sin \lambda \alpha)^{\mathrm{T}}
$$

Equation (54) or eqn (55) yields only one real root $\lambda_{1}$ with $0<\lambda_{1}<1$. The constant $c_{1}$ is related to the stress intensity factor $K_{1}$ defined by

$$
K_{\mathrm{I}}=\sqrt{2 \pi} \lim _{r \rightarrow 0} r^{1-\lambda_{1}} \sigma_{22}(r, \theta=0)
$$

with respect to the Cartesian coordinate system depicted in Fig. 1. The relation is given by

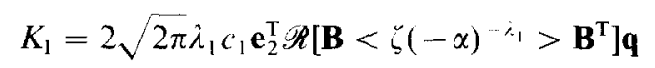

where $\mathbf{e}_{2}^{\mathrm{T}}=(0,1,0)$.

In the numerical implementation, the boundary of OABCD is discretized into $N$ line elements denoted by $C_{k}, k=1,2, \ldots, N$ with end-points $\left(x_{1}^{(k)}, x_{2}^{(k)}\right)$ and $\left(x_{1}^{(k+1)}, x_{2}^{(k+1)}\right)$. The elements are numbered consecutively in the counter-clockwise direction with $C_{1}$ and $C_{N}$ connected at the notch tip. The traction $\mathbf{t}$ and the displacement gradient $\mathbf{d}$ on tip elements $C_{1}$ and $C_{N}$ are assumed to be given by Stroh's functions approximated by $M$-term truncated series of eqn (38). $t$ or $\mathbf{d}$ is assumed constant on the remaining elements. The resulting discretized equations of eqns (29) and (30) with the mid-point of the $j$ th element chosen as the collocation points are

$$
\begin{aligned}
& \sum_{k=2}^{N-1} \mathbf{W}_{j k}^{*} \mathbf{d}^{(k)}=\sum_{k=2}^{N-1} \mathbf{U}_{i k}^{*} \mathbf{t}^{(k)}+\sum_{n=1}^{M} \boldsymbol{\Gamma}_{j n} c_{n}, j=2,3, \ldots, N-1 \\
& \sum_{k=2}^{N-1} \mathbf{W}_{k j}^{*} \mathbf{t}^{(k)}=\sum_{k=2}^{N} \mathbf{V}_{j k}^{*} \mathbf{d}^{(k)}+\sum_{n=1}^{M} \boldsymbol{\Lambda}_{j n} c_{n}, j=2,3, \ldots, N-1
\end{aligned}
$$

where $\mathbf{d}^{(k)}$ and $\mathbf{t}^{(k)}$ are the displacement gradient and traction at $C_{k}$. The analytical expressions for the matrices $\mathbf{U}_{j k}^{*}, \mathbf{V}_{j k}^{*}$, and $\mathbf{W}_{j k}^{*}$ for $j \neq k$ can be found in $\mathbf{W u}$ et al. (1992) and for $j=k$ are given by

$$
\mathbf{U}_{i j}^{*}=\mathbf{V}_{j j}^{*}=\mathbf{0}, \mathbf{W}_{j j}^{*}={ }_{2}^{1} \mathbf{I}
$$

The vectors $\boldsymbol{\Gamma}_{j n}$ and $\boldsymbol{\Lambda}_{j n}$ in eqns (59) and (60) are given by 


$$
\begin{aligned}
& \boldsymbol{\Gamma}_{j n}=\frac{1}{\pi} \mathscr{I}\left[\sum_{\kappa=1}^{3} \frac{\lambda_{n} \hat{\zeta}_{\kappa}^{(j)}}{\zeta_{\kappa}(-\alpha)^{\lambda_{n}}} \int_{z_{\kappa}^{(N)}}^{z_{\kappa}^{(2)}} \frac{\left(z_{\kappa}^{\prime}\right)^{\lambda_{n}-1}}{z_{\kappa}^{\prime}-z_{\kappa}^{(j+1 / 2)}} \mathrm{d} z_{\kappa}^{\prime} \mathbf{a}_{\kappa} \mathbf{b}_{\kappa}^{\mathrm{T}} \mathbf{q}_{n}\right] \\
& \boldsymbol{\Lambda}_{j n}=\frac{1}{\pi} \mathscr{I}\left[\sum_{\kappa=1}^{3} \frac{\lambda_{n} \hat{\zeta}_{\kappa}^{(j)}}{\zeta_{\kappa}(-\alpha)^{\lambda_{n}}} \int_{z_{\kappa}^{(N)}}^{z_{\kappa}^{(2)}} \frac{\left(z_{\kappa}^{\prime}\right)^{\lambda_{n}-1}}{z_{\kappa}^{\prime}-z_{\kappa}^{(j+1 / 2)}} \mathrm{d} z_{\kappa}^{\prime} \mathbf{b}_{\kappa} \mathbf{b}_{\kappa}^{\mathrm{T}} \mathbf{q}_{n}\right]
\end{aligned}
$$

where $z_{\kappa}^{(j+1 / 2)}=1 / 2\left(z_{\kappa}^{(j)}+z_{\kappa}^{(j+1)}\right), \quad z_{\kappa}^{(j)}=x_{1}^{(j)}+p_{\kappa} x_{2}^{(j)}$, and $\hat{\zeta}_{\kappa}^{(j)}=\cos \theta_{j}+p_{\kappa} \sin \theta_{j}$ with $\theta_{j}$ being the angle between the $j$ th element and the $x_{1}$-axis.

Additional equations of $c_{n}, \mathbf{d}^{(k)}$, and $\mathbf{t}^{(k)}$ are obtained from eqn (52) with the integration contour $\gamma$ taken as the boundary excluding the notch faces. These equations are

$$
c_{n}=2 \sum_{k=2}^{N-1}\left(\mathbf{g}_{n k}^{\mathrm{T}} \mathbf{d}^{(k)}+\mathbf{h}_{n k}^{\mathrm{T}} \mathbf{t}^{(k)}\right),
$$

where for elements on $\mathrm{OA}$ and $\mathrm{DO} \mathbf{g}_{n k}=\mathbf{h}_{n k}=\mathbf{0}$ and for those on $\mathrm{ABCD}$

$$
\begin{aligned}
& \mathbf{g}_{n k}=\sum_{\kappa=1}^{6} \frac{\zeta_{\kappa}(\alpha)^{\lambda_{n}}}{\left(1-\lambda_{n}\right) \delta_{n}^{\prime} \hat{\zeta}_{\kappa}^{(k)}}\left[\left(z_{\kappa}^{(k+1)}\right)^{1-\lambda_{n}}-\left(z_{\kappa}^{(k)}\right)^{1-\lambda_{n}}\right] \mathbf{b}_{\kappa} \mathbf{b}_{\kappa}^{\mathrm{T}} \mathbf{q}_{n} \\
& \mathbf{h}_{n k}=\sum_{\kappa=1}^{6} \frac{\zeta_{\kappa}(\alpha)^{\lambda_{n}}}{\left(1-\lambda_{n}\right) \delta_{n}^{\prime} \hat{\zeta}_{\kappa}^{(k)}}\left[\left(z_{\kappa}^{(k+1)}\right)^{1-\lambda_{n}}-\left(z_{\kappa}^{(k)}\right)^{1-\lambda_{n}}\right] \mathbf{a}_{\kappa} \mathbf{b}_{\kappa}^{\mathrm{T}} \mathbf{q}_{n}
\end{aligned}
$$

if $\lambda_{n} \neq 1$; and

$$
\begin{aligned}
& \mathbf{g}_{n k}=\sum_{\kappa=1}^{6} \frac{\zeta_{\kappa}(\alpha)^{\lambda_{n}}}{\delta_{n}^{\prime} \hat{\zeta}_{\kappa}^{(k)}} \log \left[\frac{z_{\kappa}^{(k+1)}}{z_{\kappa}^{(k)}}\right] \mathbf{b}_{\kappa} \mathbf{b}_{\kappa}^{\mathbf{T}} \mathbf{q}_{n} \\
& \mathbf{h}_{n k}=\sum_{\kappa=1}^{6} \frac{\zeta_{\kappa}(\alpha)^{\lambda_{n}}}{\delta_{n}^{\prime} \hat{\zeta}_{\kappa}^{(k)}} \log \left[\frac{z_{\kappa}^{(k+1)}}{z_{\kappa}^{(k)}}\right] \mathbf{a}_{\kappa} \mathbf{b}_{\kappa}^{\mathrm{T}} \mathbf{q}_{n}
\end{aligned}
$$

if $\lambda_{n}=1$. The factor 2 in eqn (64) is due to the fact that the plates considered are symmetric with respect to OA.

As remarked in Section 3, eqns (59) and (60) are not independent. In view of eqn (61), for traction boundary conditions specified on $C_{m}$ equations in eqn (59) with $j=m$ are selected whereas equations in eqn (60) are picked for displacement boundary conditions. The equations so obtained from eqns (59) and (60) coupled with eqn (64) lead to a set of equations with dominant diagonal elements for unknown boundary values of displacement gradients, tractions, and $c_{n}$ in the near-tip field.

To test the accuracy of the numerical method, a plate of $h / w=1$ containing a single edge crack of length $a$, or two collinear edge cracks of length $a$, or an internal crack of length $2 a$ was considered. In the numerical calculations it was found sufficient to discretize each side of OABCD into 10 elements of equal length and to use two eigenmodes to represent the near-tip field. Results with the number of elements doubled were also calculated. The changes in the values of the stress intensity factor are less than $1 \%$. The values of $K_{\mathrm{I}}$ normalized by $\sigma_{0} \sqrt{\pi a}$ for $\beta^{2}=10,2.5,2,1,0.5,0.1$ as $a / w=0.3,0.4,0.5$ are given in Table 
Table 1. $K_{1}$ normalized by $\sigma_{0} \sqrt{\pi a}$ for a single edge cracked plate (S), a double edge cracked plate (D), and an internally cracked plate (I)

\begin{tabular}{cccccccccc}
\hline & \multicolumn{3}{c}{$a / w=0.3$} & \multicolumn{3}{c}{$a / w=0.4$} & \multicolumn{3}{c}{$a / w=0.5$} \\
$\beta^{2}$ & $S$ & $D$ & $I$ & $S$ & $D$ & $I$ & $S$ & $D$ & $I$ \\
\hline 10 & 1.70 & 1.12 & 1.07 & 2.11 & 1.14 & 1.12 & 2.79 & 1.18 & 1.19 \\
5 & 1.70 & 1.14 & 1.08 & 2.11 & 1.16 & 1.13 & 2.79 & 1.20 & 1.21 \\
2 & 1.70 & 1.18 & 1.10 & 2.11 & 1.21 & 1.16 & 2.79 & 1.24 & 1.26 \\
1 & 1.69 & 1.24 & 1.13 & 2.11 & 1.28 & 1.22 & 2.79 & 1.32 & 1.33 \\
0.5 & 1.69 & 1.33 & 1.18 & 2.11 & 1.40 & 1.29 & 2.77 & 1.46 & 1.44 \\
0.3 & 1.70 & 1.41 & 1.22 & 2.11 & 1.52 & 1.36 & 2.77 & 1.60 & 1.54 \\
0.2 & 1.71 & 1.49 & 1.26 & 2.13 & 1.63 & 1.43 & 2.77 & 1.74 & 1.64 \\
0.1 & 1.77 & 1.62 & 1.35 & 2.18 & 1.82 & 1.55 & 2.79 & 1.99 & 1.81 \\
\hline
\end{tabular}

Table 2. $K_{1}$ normalized by $\sigma_{0} w^{\prime} i_{1}$ for a single edge notched plate $(\mathrm{S})$ and a double edge notched plate $(D)$

\begin{tabular}{ccccccccc}
\hline & \multicolumn{3}{c}{$a / w=0.3$} & \multicolumn{2}{c}{$a / w=0.4$} & \multicolumn{2}{c}{$a / w=0.5$} \\
$\beta$ & $\lambda_{1}$ & $S$ & $D$ & $S$ & $D$ & $S$ & $D$ \\
\hline 10 & 0.605 & 2.41 & 1.60 & 3.37 & 1.84 & 4.84 & 2.09 \\
5 & 0.587 & 2.30 & 1.53 & 3.22 & 1.76 & 4.67 & 2.00 \\
2 & 0.562 & 2.12 & 1.43 & 3.02 & 1.65 & 4.42 & 1.88 \\
1 & 0.544 & 2.00 & 1.35 & 2.88 & 1.57 & 4.29 & 1.79 \\
0.5 & 0.530 & 1.88 & 1.32 & 2.72 & 1.54 & 4.07 & 1.77 \\
0.3 & 0.522 & 1.81 & 1.32 & 2.64 & 1.56 & 3.97 & 1.79 \\
0.2 & 0.518 & 1.77 & 1.33 & 2.58 & 1.60 & 3.90 & 1.85 \\
0.1 & 0.511 & 1.73 & 1.40 & 2.54 & 1.73 & 3.83 & 2.04 \\
\hline
\end{tabular}

1. The values for the isotropic plate agree well with those reported by Rooke and Cartwright (1976). The values of $K_{\mathrm{I}}$ for internal crack for all $\beta$ values considered are in close agreement with those listed in (Sih, 1973). It is interesting to note that for single edge crack the $K_{1}$ is almost constant as $\beta^{2}>0.5$ for the crack lengths considered. For double edge or internal cracks, $K_{\mathrm{I}}$ approaches to a constant value as $\beta$ increases.

Consider next the V-notched plates. The problem for isotropic single notched plate has been done by Gross and Mendelson (1972) by eigenfunction expansion method. Here, a plate of $h / w=1.4$ containing a single notch or two symmetric notches with $\alpha=3 \pi / 4$ is investigated. In the numerical computations, AB was divided into 10 equal-sized elements, $\mathrm{BC}$ and $\mathrm{CD}$ into five elements each, and $\mathrm{OA}$ and $\mathrm{OD}$ into elements of length about $0.1 w$. The values of $K_{1}$ nondimensionalized by $\sigma_{0} w^{1-\lambda_{1}}$ for $\beta^{2}=10,2.5,2,1,0.5,0.1$ as $a / w=0.3$, $0.4,0.5$ are given in Table 2. For isotropic single V-notched plates, the values of $K_{\mathrm{I}}$ obtained for single notch agree closely with those in Gross and Mendelson (1972). For single notch, $K_{\mathrm{I}}$ varies in the same trend as $\beta$ or $\lambda_{1}$; while for double notches, there appear local minima in the neighborhood of $\beta^{2}=0.5$.

In addition to the stress intensity factors, the numerical results contain either tractions or displacement gradients on the mid-points of the regular boundary elements. Presented in Figs 3 and 4 are the distributions of $\sigma_{22}$ ahead of the tip for isotropic single and double $\mathrm{V}$-notched plates for $a / w=0.4$, respectively. For comparison purposes, the results calculated by one-term and two-term eigenfunction expansion were also plotted in Figs 3 and 4 . Figure 3 shows that the values given by the regular elements vary almost linearly, indicating a dominant bending effect, and are consistent with the two-term approximation up to $r / w=0.25$ where $r$ is the distance from the tip. Figure 4 reveals that $\sigma_{22}$ on the regular elements approaches a constant value away from the notch tip and is consistent with the two-term approximation over the whole range.

\section{CONCLUDING REMARKS}

A complex-variable boundary element method for notched anisotropic bodies is presented. In the proposed method analytic structure of the near-tip field is included so that 


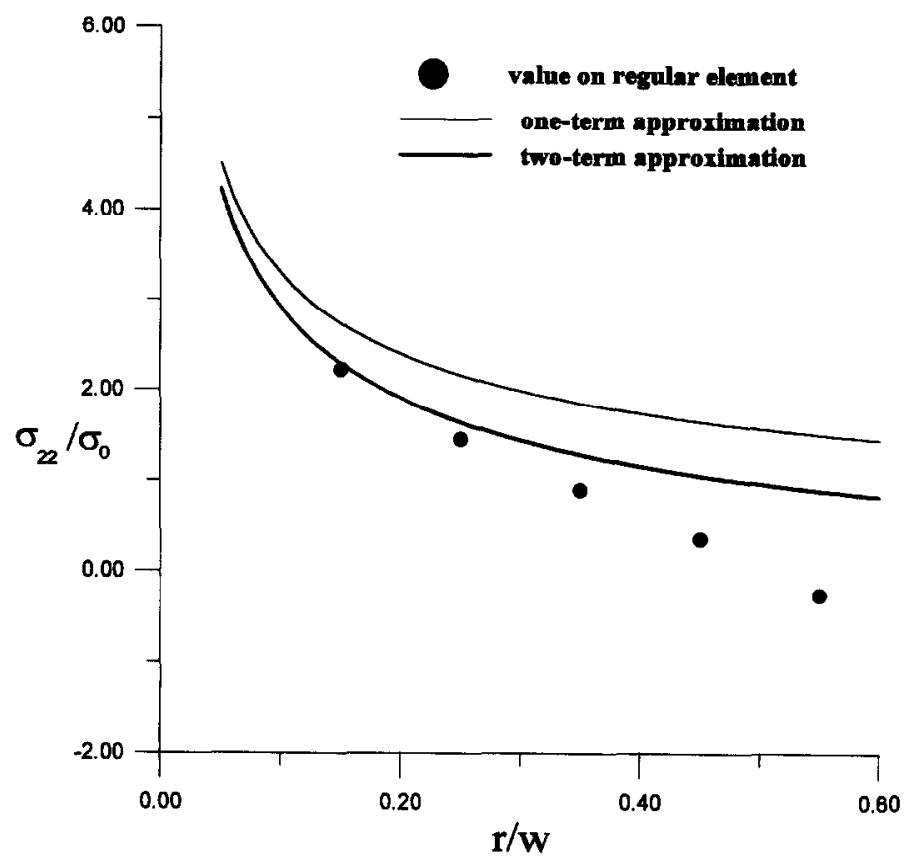

Fig. 3. Distribution of $\sigma_{22}$ ahead of the tip for isotropic single V-notched plate.

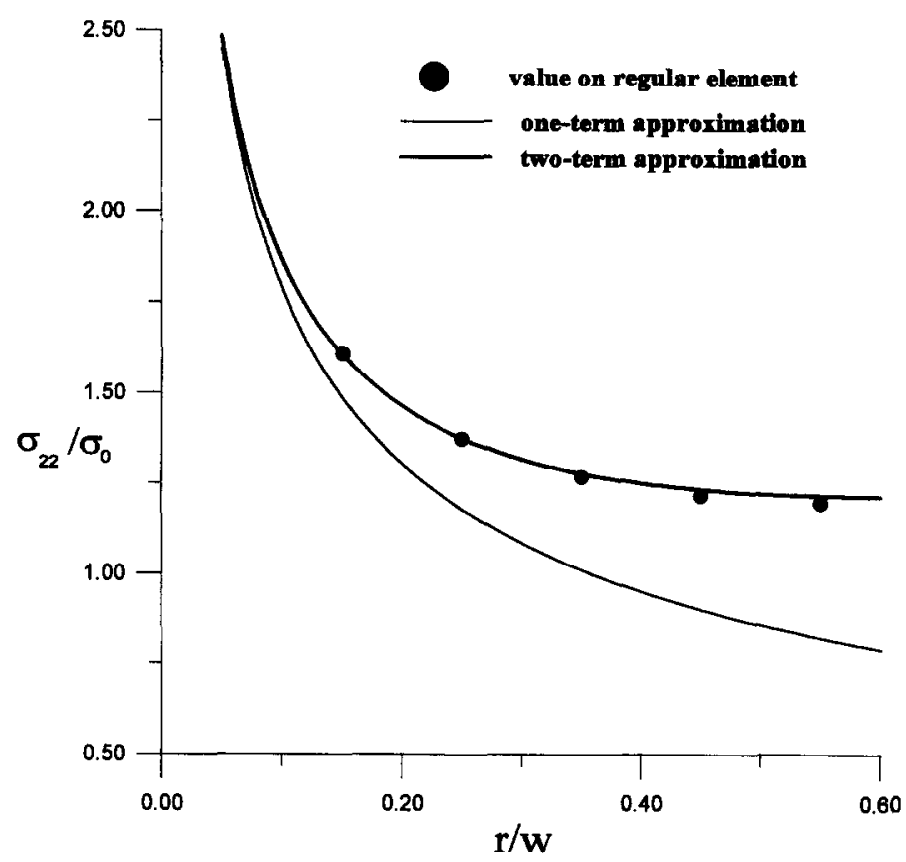

Fig. 4. Distribution of $\sigma_{22}$ ahead of the tip for isotropic double V-notched plate.

$K_{1}$ can be obtained directly without post-processing. The method is efficient in that accurate results can be obtained by relatively few constant boundary elements and a limited number of eigenfunctions at tip elements.

The proposed method is used to investigate notched plates of special orthotropic material under uniform tensile stress. It is found that for single edge crack the $K_{\mathrm{I}}$ is almost constant as $\beta^{2}>0.5$. It is also found that for single notch of notch angle $3 / 2 \pi$, the SIF's vary in the same trend as $\beta$ or $\lambda_{1}$; while for double notches, there appear local minima in the neighborhood of $\beta^{2}=0.5$. 
Acknowledgement - I his research was supported by the National Science Foundation of the Kepublic of China, under grant No. NSC 82-0401-E-002-089.

\section{REFERENCES}

Barone, M. R. and Robinson, A. R. (1972). Determination of elastic stresses at notches and corners by integral equations. Int. J. Solids Structures 8, 1319-1338.

Dongye, C. and Ting, T. C. T. (1989). Explicit expressions of Barnett-Lothe tensors and their associated tensors for orthotropic materials. Q. Appl. Math. 47, 723-734.

Eshelby, J. D., Read, W. T. and Shockley, W. (1953). Anisotropic elasticity with applications to dislocation theory. Acta Metall. 1, 251-259.

Gross, B. and Mendelson, A. (1972), Plane elastostatic analysis of V-notched plates. Int. J. Fract. Mech. 8, 267276.

Rizzo, R. (1967) An integral equation approach to boundary value problems in classical elastostatics. $Q . J$. Appl. Math. 25, 83-95.

Rooke, D. P. and Cartwright, D. J. (1976). Compedium of Stress Intensity Factors. HMSO, London.

Sih, G. C. (1973). Handbook of Stress Intensity Factors. Institute of Fracture and Solid Mechanics, Lehigh University, Bethlehenl.

Stroh, A. N. (1958). Dislocations and cracks in anisotropic elasticity. Philos. Mag. 7, 625-646.

Ting, T. C. T. (1995). Generalized Dundurs constants for anisotropic bimaterials. Int. J. Solids Structures 32 $(3 / 4), 483-550$.

Ting, T. C. T. and Chou S. C.. (1981). Edge singularities in anisotropic composites. Int. J. Solids Structure. 17 (11), 1057-1068.

Wu, K.-C. (1989). Representation of stress intensity factors by path-independent integrals. J. Appl. Mech. 56, $780-785$.

Wu, K.-C. and Chang, F.-T. (1993). Near-tip fields in a notched body with dislocations and body forces. $A S M E$ J. Appl. Mech. 60, 936-941.

Wu, K.-C., Chiu Y.-T. and Hwu, Z.-H. (1992). A new boundary integral equation formulation for linear elastic solids. ASME J. Appl. Mech. 59, 344-348. 